\title{
Spatial Thinking With Comics in Geography Education
}

\section{Frederik von Reumont* and Alexandra Budke}

Institute of Geography Education, Faculty of Mathematics and Natural Sciences, University of Cologne, Cologne, Germany

Spatial thinking is a key asset in geography education. Thus pre-service geography teachers need to be trained in spatial thinking. Since comics seem to be a good approach to teaching geography, in our study we wanted to find out about the possibilities to teach spatial thinking with comics. How do students put their spatial thinking into practice while producing their own comics? What does this mean for the learning process? We analyzed comics, which were produced by teacher students in a mid-level university seminar for the use in the geography classroom. In the analysis of the comics produced by our students we gauged their abilities to think spatially in terms of the spatial concepts developed by Wardenga. We contextualized the visual output of the comics with a survey we conducted among the participants, which we analyzed using qualitative content analysis. We found that our students had difficulties assessing the potential of comics to teach spatial thinking in a purely verbal and theoretical way. However, in their own productions they showed high levels of creativity expressing spatial concepts. Comics produced by learners offer unique opportunities for the reflection and assessment of their ways of spatial thinking.

Keywords: spatial thinking, comics, creativity, creative learning, geography education, teacher training, spatial concepts

\section{INTRODUCTION}

Teachers who use comics as part of classroom-based learning often hope that these comics will help teach students complicated or abstract topics (Lin et al., 2015) such as physics, history, literature, or chemistry. The aim of using comics is to motivate students to engage with a topic, which may be made easier to understand in comic form and is associated with fun or well suited to young readers who consume comics in their free time. The combination of pictures and conversational language in comics can help students to enjoy learning about science (Lin et al., 2015) or historical facts (Munier 2000) while engaging in an interesting narrative. The popularity of comics as classroom material is increasing, with more and more comics being published specifically as a learning resource for teaching science, such as the comic World of Viruses for biology classes. However, these learningspecific comics do not always have to be strictly scientific. Tatalovic (2009) stated that science comics can make use of fictitious settings for their explanations of the real world. Or conversely even fictional scenarios, such as those in super hero stories, can very well deliver "hard" scientific facts.

Gomez (2014) and Kleeman (2006) emphasize the suitability of comics for introducing sensitive and complex topics. In particular, the use of humor can reduce the aversion of many learners to engaging with complicated topics. Gomez (2014) argued that comics are a tool for revealing differing perspectives on geographical phenomena, such as natural disasters. He argues that comics, with their emphasis on action, can put learners in a position where they can appreciate and discuss geographical 
content in the context of its application. Kleeman (2006), too, stressed the inherent incentive of comics to encourage critical thinking that can subsequently be applied to geographical topics.

Most studies rely on professionally produced comics. Researchers have focused their attention on passive learning activities, in which learners consume comics. However, comics have an overwhelming potential for creative production when authored by the learners themselves. Lay (2021), for instance, reports on a practical teaching experience in a project carried out by Germany's cultural institute, the Goethe Institute, where participants drew chapters of a story for learning German. Kilıçkaya and Krajka (2012) carried out an empirical study on Turkish speaking students learning English. Both agree on the motivational effects on young learners of creating comics as learning method. Additionally, Lay (2021) points out that learners will thereby generate a deeper understanding of visual media and multimodal texts in general. Other studies corroborate the positive value of this method, e.g. in the fields of management and tourism, where Silva et al. (2017) and Elsayed and Daif (2019) observed high levels of creativity in the learners, who also developed critical thinking skills and practiced reflection in the process. Dallacqua and Peralta (2019) report on their experience of teaching science with comics created by the learners. They call attention to the active role learners take on, making them selforganize their meaning making about the world around them. A key asset for understanding geography is spatial thinking. To our knowledge, studies examining the creation of comics for learning geography and promoting spatial thinking do not yet exist.

Comics create narratives through the combination of text and pictures and the arrangement of these elements in the space of the page. These three aspects offer a wide range of creativity. All the opportunities a movie director has to stage a story also offer themselves to the comic creator, starting with character design, camera positioning, choice of location, etc. This wealth of choices offers a great playground for creativity to the comic creator, because in contrast to film, the comic creator can work alone. All one needs is a pen and paper. That is why comics seem to be a very good means of integrating creativity into teacher training at university. Incidentally, students can take the opportunity to assess the method for their own future use in the classroom, while working with it themselves.

In this paper we present a study in which we examined the creative process of producing geographical comics with 32 undergraduate students at university level enrolled in a geography teacher training course at the University of Cologne, Germany. The study aims to explore how learning by creating comics affects spatial thinking in geography students. The primary aspect of geography is to scrutinize all phenomena related to space and spatial relations on the earth's surface. The standards for German geography education stress the importance of spatial thinking, which is essential for any further understanding of geographical meaning making (Deutsche Gesellschaft für Geographie (DGfG), 2014). The capacity to think spatially is crucial to the education of geographers and geography teachers alike. To formalize the idea of spatial thinking, four concepts of space were introduced into German geography education by Wardenga
(2002); see also Spatial Thinking in the Context of Comics Section).

In our study, we first wanted to assess whether our teacher students at university are able to exploit the possibilities of comics for teaching and learning spatial thinking. Then we were interested in how they planned to translate their own spatial thinking into the comic form. Lastly, we wanted to find out how they put their understanding of conceptualized space into practice in comic production. We compared their practical ideas expressed in questionnaires prior to comic production with their concrete realizations in the verbal and non-verbal forms of their finished comics. We worded our research question as follows:

- How do students put their spatial thinking into practice while producing their own comics?

When assessing the possibilities of learning spatial thinking through comics, we first have to ensure that they can express or represent space in a geographically meaningful way. The possibilities of comics to do this are outlined in the following section. We will then describe the design and methods of our study. Subsequently we present and discuss our findings. The paper concludes with suggestions for teaching geography by letting the students create their own comics.

\section{SPATIAL THINKING IN THE CONTEXT OF COMICS}

Spatial thinking in the US is rooted in the neurological findings which ascribe certain "hard-wired" capabilities to the human brain, allowing it to grasp space in distinct modes (Gersmehl and Gersmehl, 2007, p. 188). Anthamatten (2010), p. 169), citing the US National Academy of Sciences, points out that spatial thinking "in terms of power and pervasiveness (...) is on par with (...) mathematical or verbal thinking." He further emphasizes the need to "codify [spatial thinking] in the form of basic spatial concepts" (ibid.) to make it available for a "distinctive form of reasoning" (ibid.). Rhida et al. (2020) point out the importance of spatial thinking in the context of modern technology like GIS. They particularly stress the "process of reasoning" (p. 2) and the conceptualization of space as essential parts of spatial thinking. Modern geography education in Germany utilizes four such concepts to answer geographical questions. Differing from the US American approach to spatial thinking, the German term "Raum" includes a whole array of spatial ideas ranging from the realist approach to the constructivist world view, each perspective describing space in terms of differing premises and axioms. The four concepts of space aim at covering all geographically relevant interpretations of the term. Wardenga (2002) originally developed this system of concepts for German geography school curricula, which now define the understanding of space in the national standards of German geography education (Deutsche Gesellschaft für Geographie (DGfG), 2014). The first concept describes space as (self-) contained (Wardenga, 2002), which corresponds to a view broadly adopted by the 
natural sciences. Geographical phenomena are treated as independently existing entities, ready for description by means of measurement and quantification in an enclosed physical space. This also applies to the second concept, which describes space as spatial relations in physical spaces (Wardenga, 2002). It differs from the container space by focusing on the locations and interconnections of phenomena in space. This is a profoundly geographic way of seeing the world and is arguably spread across the whole discipline throughout the world. Both of the aforementioned concepts consider space as objective. They aim to stimulate in students an understanding of space that helps to explain real spatial phenomena like in the natural and social sciences.

The third concept is concerned with how perceived space is comparable to psycho-geographical approaches that attempt to examine the embodied experience of space (Wardenga, 2002). The fourth concept, interpreting space as a social construction, is informed by constructivist ideas. This conception of space focuses on the production of space through social communication in the text form, which has been prominent in critical approaches to geography and cartography in Anglophone research communities.

There are numerous ways geographers think and talk about space. However, visual or graphic representations tend to play a vital role in the process (Elbay, 2020). In the next section we will theoretically determine whether comics have the capacities to express or represent space in geographically relevant ways as a prerequisite for working with them in a geographical context and using them for spatial thinking. We will apply the four concepts of space described above to assess the capacities of comics for spatial thinking. In the analysis of the comics produced by our students we will gauge their abilities to think spatially in terms of these concepts as well.

\section{Space as (Self-) Contained Space in Comics}

Spaces can be considered containers literally containing geographically relevant phenomena and objects. Spaces collect different geographical phenomena that exist in a shared space independently of each other (Wardenga, 2002). When space is examined under this premise, we can describe contained objects as singular entities that are part of a physical-material world. This requires a description of the characteristics shaping the landscape that is as exact as possible, e.g., the landforms as processual results of natural forces, infrastructure and buildings as results of human action, etc., The points of view of individuals or social groups are not crucial, but the goal is a factual presentation that should be as objective as possible.

An authentic description is essential for the interpretation of space as self-contained space. The trans- or multimodal nature of comics allows for the incorporation of media such as diagrams, charts, and maps, which serve as the bases for assertions and can support a scientific mode of presentation. Embedded in attractive artwork, alongside an inspiring story line, such comics may motivate young learners to approach difficult geographical issues.

Authors commonly draw the pictures from photographic references to achieve a high level of detail, in order to achieve credibility and plausibility. A meticulous reproduction of even minor details of the environment in a scene reproduced from photographs can enhance the impression of picturing real scenery. This technique is often used in comic journalism, using photos or sketches from the field as documents of reality. The resulting images appear factual. Integrating a diagram or other infographics can further underline the factuality of the comic's assertions. This seamless inclusion of different visual means of expression, such as diagrams, charts or maps, into the narrative, is typical of a montage or collage in science comics. In this way the creators of the comic can insert assertions about the narrative into the comic itself, emphasizing objectivity, and the whole comic page can thereby turn into a diagram.

To "read" the images correctly, the audience often needs a certain amount of previously acquired knowledge in order to decode the graphical schemas, e.g. identifying the design of characters (hairdo, clothing etc.), models of vehicles, types of buildings, or the behavior of protagonists. In many cases, aspects like these indicate a belonging to certain locations on the globe. The deployment of these stereotypical symbols alludes to socially constructed imaginations of space in order to create shortcuts for the flow of information. This shows the limits of objectivity of pictures endowed with any kind of meaning or functionality, even if photographic references or the photographs themselves were used.

Even in the most factual comics there is no way around subjective choices which might themselves be socially influenced. However, comics allow us to address real world issues, as many comic journalists, such as Joe Sacco, have demonstrated. They use comics to make the subjective influences on factual reporting transparent. For the purposes of this study, it suffices that the comics constitute an attempt at scientific correctness, because in geography education we deal with real world phenomena such as migration or tourism. Whether or not "objectivity" is achieved is not important, but rather how the participants of this study try to achieve it.

\section{Space as Spatial Relations in Comics}

If space is reflected as spatial relations between objects, the closed space of the container opens up to reveal a continuous network of spatial relations. Here, space is defined by spatial relations, their distributions in space, and their patterns of interconnections (Wardenga, 2002). The descriptions of space as spatial relations in comics should also be mainly realistic, aiming at objectivity in a scientific way. A valid depiction of spatial relations in a comic has to show two or more objects simultaneously and demonstrate their interrelationship.

In comics, the composition of the whole page is based upon the arrangement of its panels, which offers creators the opportunity to position panels according to the geographical locations displayed on the page. In comics the order of the panels on the page does not have to follow a rigid order, zig-zagging in reading direction across the page, like words or sentences in text or even frames in film. The panels may rather adhere to specific locations where-not when-the action they display takes place. For this purpose the backdrop image of scenery may function as a kind of map, providing spatial orientation. When the panels are 
connected across this background, e.g., by arrows, they create a network of interrelated phenomena. The result is a comprehensive visualization of a complex set of spatial relationships. This is possible because not only sequences but also spatial arrangements of panels carry meaning in the construal of the narrative ( Witek, 2009).

Many examples of comics demonstrate that comics' depictions of space are not confined to showing closed container-like spaces just because they are bound by panel or page borders. Speech bubbles can point to characters outside the panel, invisible to the reader. The direction of a character's gaze or gestures, and the orientation of objects or characters can be a reference to objects or phenomena in an adjacent panel, explaining their spatial relations (Witek, 2009). Furthermore, maps inserted in the comic often refer to real or imagined places outside the space of the panel.

\section{Space as Perceived Space in Comics}

Geographical space is perceived by individuals, each living within their own spatial perceptions, assessing them according to their own personal experiences and acting accordingly. The analysis of differing perceptions of space by various stakeholders may help to single out reasons for conflicts concerning the usage of certain places. Such analyses can provide various perspectives on and appraisals of places for geography students (Wardenga, 2002). Creators of comics can show emotions and individual sensory impressions as a result of subjective perceptions of space. This interpretation of an environment can result in emotional landscapes representing space as it is perceived by an individual.

Color can play an important role in the depiction of subjectively perceived space in comics. Particular palettes can be used to express atmospheric moods describing the emotional life of the protagonists and mirroring their personal relationships with the surrounding space. The artist can achieve a complete description of three-dimensional space solely by using color and color values, a technique called atmospheric perspective. It is assumed that the further away an object is located from the observer, the more it loses its sharp contours and color saturation (Schüwer, 2008). The artist turns this color palette into a metaphorical atmosphere expressing the emotional landscapes perceived by the protagonist. The expressionist atmospheric perspective is bound to the position (in a literal as well as in a figurative sense of the word) of the protagonist.

In comics the creators can also use the geometrical construction of perspective to stage emotions existing at this particular location, e.g., with high or low-angle "camera shots". For instance, when the camera looks up to a person, this perspective lets this character appear to be in control and determined, maybe even cruel, standing above the others, a commonly used mode of expression in both comics and film (Mateu-Mestro, 2010).

\section{Space as Social Construction in Comics}

In social communication, space is expressed as a social construction (Wardenga, 2002). We have all heard of "trendy neighborhoods" or "no-go areas". We use these concepts of space to form an image of what to expect and how to judge these spaces, replacing or influencing firsthand experience. This means that we perceive or imagine certain spaces under the influence of what we have read or heard about them. Conversely, city planners might want to influence productions of space for city marketing, using techniques of PR to spread positive images of their city. Different ideas of places usually influence each other and are thus a collective imagination of space. In constructivist terms this means that by producing and reproducing these ideas we produce space itself. Comics are an effective tool for describing collectively constructed images of space with the use of symbols that are generally accepted or well-known in a specific target group.

Comics can draw on a wide variety of well-known symbols and clichés to represent space as social construction. In comics the creators often take advantage of the fact that they can describe places with the help of certain objects that are associated with a particular place in the recipient's imaginations. For instance, famous buildings like the Eiffel Tower or other objects are often displayed, hinting as to the location of a scene (Lefèvre, 2009). Plants, animals, food, stamps, traditional clothing, hair styles, or cars can fulfil the same function if their association with a location is strong enough. The depiction of festivals, fairs, or rites can serve the same purpose. When Donald Duck travels to Cologne, of course it is Carnival season, as seen in The Ducks in Germany (Disney Enterprises, 2015). Even letters from certain alphabets can indicate location, e.g. the display of Chinese characters on street signs might place a scene in China. For such a use of symbols the audience has to be able to identify the symbols and assign them to the right places, and therefore requires a certain degree of previous knowledge to correctly locate a story. Additional text can be used to explain the symbolism and help to guide the audience's interpretation.

This review of some of the structural and stylistic means used in comics in the light of four geographical conceptualizations of space clearly demonstrates that comics fulfil the prerequisites to make geographically relevant statements about space. In the following we will outline our study, which assessed the preparedness of students studying to become geography teachers for using comics for teaching. Additionally, we will discuss our methods of showing whether the production of comics can promote spatial thinking in our students and how we as educators can achieve deep insights into the understanding of space through the analysis of students' comics.

\section{METHODS}

We conducted our study within a seminar about innovative teaching methods. We designed a seminar where students had to create their own comics as geography teaching materials for school children. In the study we wanted to assess the ability of university students studying geography education to use the language of comics for spatial thinking before and after a short intervention about different concepts of space. In our research design we used not only questionnaires to assess the students' opinions in a verbal form, but we also looked at their sketches and their finished comics to reveal a deeper understanding of their ideas of spatial thinking. 


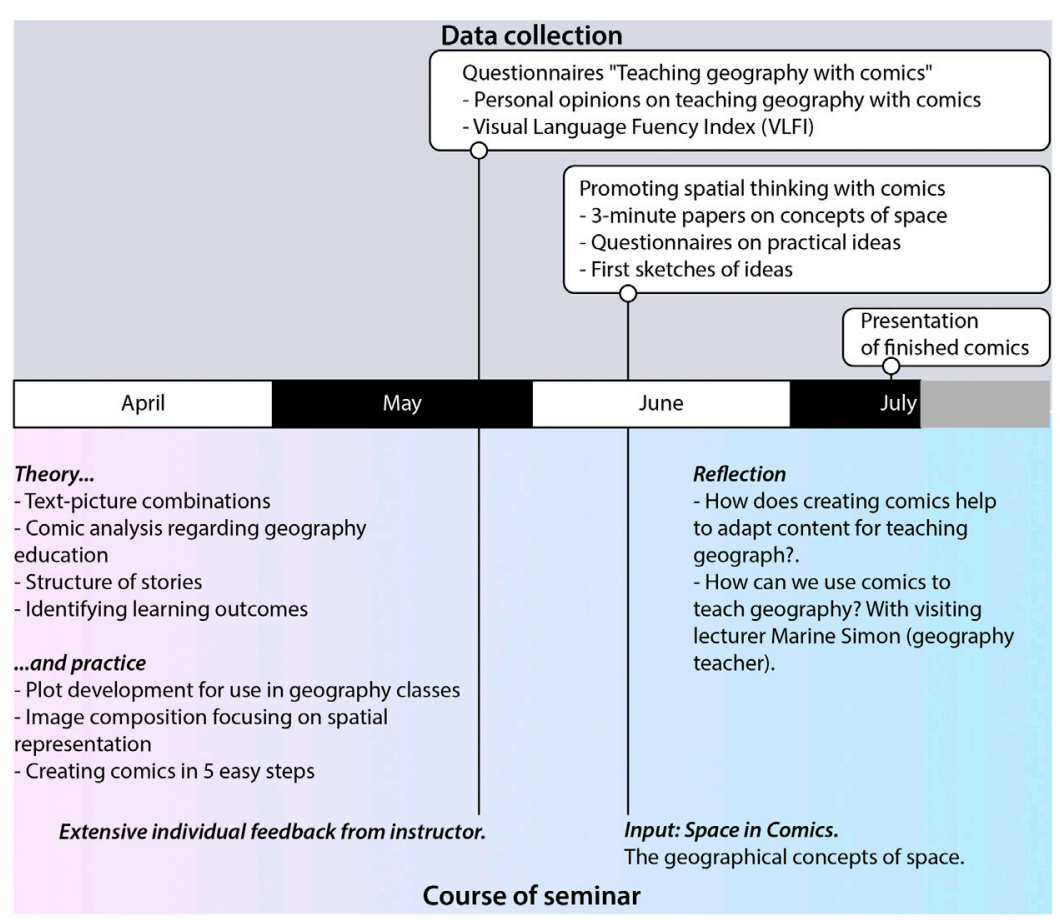

FIGURE 1 | Timeline of the course of the seminar and data collection for this study.

\section{The Participants}

The participants in the study were the 32 participants in the seminar about innovative learning methods in geography education at the University of Cologne, Germany. All students except for one were enrolled in their 4th to 6th semester of the Bachelor's degree program, studying geography education for secondary education (grades 5-10). There were 13 males and 19 females. Their interest and expertise in comics was low: 6.68 on the Visual Language Fluency Index (Cohn's VLFI, Cohn 2014). The VLFI can be used as a measure of a person's expertise in comics. It is strongly based on the assessment of media consumption like comic books, comic strips, or graphic novels. We can thus also interpret it as a measure of interest in comics. A score of 8 or less can be considered low visual language fluency. Cohn (2014) considers scores between 10 and 19 points to be average values. A high fluency level starts at 20 points. 24 students scored less than eight points, 7 even less than four points.

\section{Procedure: Training Concepts of Space with Extracts From Comics}

Our study was an integrated part of a seminar for teacher trainees at university level in a bachelor's degree course (Figure 1). Within the scope of the seminar, the students had to produce comics which are suitable for teaching geography. The first half of the seminar was dedicated to the theory of comics, starting with storytelling techniques, the combination of text and image, panel arrangements, and image composition, all in relation to science comics discussing geographical problems. The analysis of existing comics about geographical problems was also part of this thematic block. Towards the middle of the semester we conducted a survey among the students to assess their attitudes towards teaching geography with comics. In the subsequent session a brainstorming exercise activated the students' previous knowledge about the concepts of space in German geography education, as detailed in Spatial Thinking in the Context of Comics Section. The results of this exercise are documented in very short written papers by each student (3-min papers, $n=29$ ). We then gave a lecture aiming at recalling the said concepts of space and what they might mean in the context of comics (Figure 1). This was accompanied by four example pages from previously existing comics, showing each of the four concepts in a particularly clear way. Afterwards the participants had to choose one or more of the four concepts for their own story ideas, which they had developed during the course of the seminar. They had to give reasons for their choices and state how they would realize the concepts in their comics.

The two surveys, the sketches, and the finished comics provided us with insights into how creating comics has impacted on the participants' understanding of the concepts of space.

\section{Questionnaires for Assessing the Future Teachers' Attitudes Towards Comics}

After having several sessions of the seminar dedicated to basic knowledge of comics, we wanted to find out more about the group's $(n=31)$ general attitudes towards teaching geography with comics and whether or not they were able to appreciate the possibilities of comics for spatial thinking in geography classes. We handed out questionnaires consisting of Likert scales and open questions to be answered with short sentences. In the questionnaires, we first asked the participants whether comics are suitable for geography education in general. We went on to 
ask whether the participants found comics suitable 1) for promoting spatial thinking in learners, and 2) for conveying the four concepts of space to young learners as required by the curriculum. After directing attention to the aspect of space in comics, we designed the last question to evaluate the general use of comics in teaching geography again by asking the participants whether they themselves would actually consider the use of comics in their own future classrooms. For the analysis of the written records transcribed from the questionnaires we used the technique of content analysis introduced by Mayring (2014). We used inductive category formation, establishing a code for each relevant statement while browsing through the texts. Whenever a statement matched an already existing code, we marked it accordingly. In this way we could count the number of occurrences of the various codes in the answers provided by the students. The lowest unit for establishing a code was the single word. After working through all the texts, we summarized the codes into higher order categories wherever possible.

\section{Assessing the Participants' Ways of Spatial Thinking}

Having acquired knowledge about the concepts of space in the intervention, the next task for the participants was to form their own ideas of how to apply this knowledge to their comic projects. For the purposes of subsequent analysis the participants documented this planning phase of their comics. In this way we could compare their concrete action with their verbally expressed opinions from before the intervention.

The first step for them was to choose one or more of the concepts of space for their comic stories. This meant that the participants had to assess, in a first step, which of the concepts were most suitable for their own stories. Then they had to explain the reasons for their choices. In the next step they had to apply their knowledge of the spatial concepts to their individual stories. The last step, in particular, left room for creativity: they were supposed to make a sketch of the key scene of their comic, outlining the spatial concept of their choice. This was meant to help the students develop their stories while being mindful of spatial thinking.

We analyzed the written answers to the open questions in a similar way to the first survey, using the technique of thematic analysis as proposed by Mayring (2014). Thematic analysis is a method combining inductive and deductive category formation for the qualitative analysis of students' statements. First we assigned each statement from the questionnaires to the concept of space chosen by the participants. We sorted the students' answers into the categories of space as container, space as spatial relations, perceived space, and space as social construct. We further classified the answers according to whether they gave reasons for the choice of concept or whether they explained the stylistic means with which the students planned to realize the spatial concepts in their comics. Finally, we coded the statements, subsuming them into categories inductively. Afterwards we counted the occurrences of each sub-category to see whether there was a striking number of certain categories. This thematic analysis helped us to evaluate the participants' reasons and ideas for the use of spatial concepts in their comics.

\section{Analyzing Spatial Thinking: A Multimodal Approach With Comics}

For the analysis of the participants' spatial thinking we used their preliminary sketches as well as their finished comics. At the point of collecting the data for this study we asked them to make a sketch of a key scene of their comics illustrating their use of the chosen concepts of space. The participants had already created plots for their comic projects. This way we ensured that from the beginning we took into account not only the participants' verbal expressions, but also their image-based imaginations of space. We wanted to tap into the visual channel of the students' ways of spatial thinking and, as a learning outcome for the participants, encourage them to plan their comics using visuals. We collected the finished comics at the end of the term and used them together with the sketches for the analysis of the students' ways of spatial thinking and how making comics had left its mark on them.

The analysis of comics is a fairly demanding task, because of comics' innate multimodality, using both text and imagery in a multitude of panels arranged in a meaningful order. For the analysis we took a look at the content of the story as a whole, to identify the participants' general approach to tackling a given geographical problem. It is possible to address a single problem through the lens of different spatial concepts. We can consider the questions of mass tourism, for instance, by individual perceptions of hotspots like beaches. Another approach could be to examine the same phenomenon considering aspects of the social constructedness of marketing concepts for certain travel destinations, or the measurable effects of mass tourism on specific locations. All of these approaches deploy different conceptions of space. Within a narration these concepts may change or mix. So in a next step, we identified the single key scenes of the comics, as indicated in the questionnaires, for which the participants had already made sketches. We examined whether these scenes fit the declared intentions the participants had expressed in the questionnaires. The participants also provided their own reasoning for their choices, offering insights into their conceptual understanding of space on a purely verbal level. Comparing these thoughts about the concepts of space with their concrete interpretations gave us very deep insights into their ways of spatial thinking. The fact that they expressed themselves with comics meant that they had to completely rethink in a multimodal way what they had expressed earlier purely verbally. The result is not a reiteration but a nontrivial extension of the description of space through the eyes of the participants. Guiding questions for the analysis of the key scenes concerned the content of the story and how it related to the chosen concept of space. In a further step we examined how the participants' storytelling with text and pictures supported the concept of space chosen by the participants. We focused on the stylistic devices used to convey the concept of space. We considered aspects such as the use of speech or thought bubbles, the page layout, panel composition, and character design, but of course our main interest lay in the depiction of space. We focused on the use of perspective, the placement of characters and other objects within the space of the story world, textual descriptions or annotations, and the general use of stylistic 


\section{Comics are suitable for teaching...}

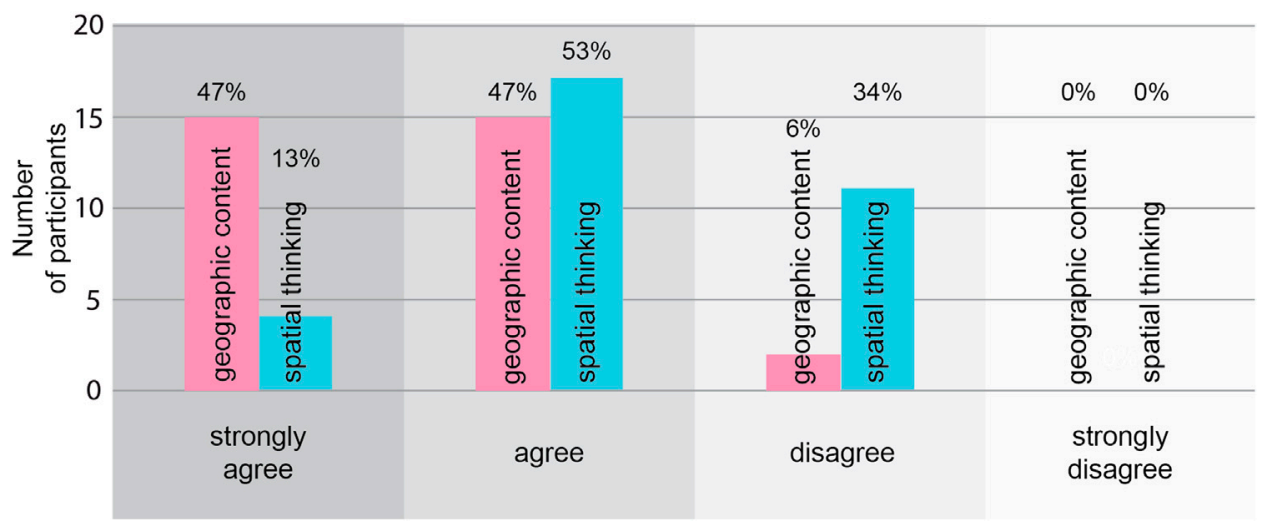

FIGURE 2 | Attitudes of Geography teacher trainees towards teaching Geography with comics on a Likert scale $(n=32)$

devices like the use of color, brush stroke, and so forth to scrutinize the participants' palette of visual devices for expressing spatial thinking. The results of these analyses were brought together by evaluating whether content and style supported each other in conveying the concept of space chosen by the students. This enabled us to effectively draw conclusions about the students' understanding of space regarding the concepts required by the German national standards for geography education.

\section{RESULTS: HOW DO STUDENTS PUT THEIR UNDERSTANDING OF CONCEPTUALIZED SPACE INTO PRACTICE IN COMIC PRODUCTION?}

Although initially our students had difficulties linking comics with spatial thinking, in their practical work they demonstrated, that they were able to creatively exploit the visuo-verbal language of comics to express their spatial thinking. This provided deep insights into their thought processes. The finished comics offer rich opportunities for a guided reflection and thorough understanding of conceptualized space.

\section{How do Students Judge the use of Comics for Learning Spatial Thinking?}

When asked about teaching geographic content with comics most students displayed positive attitudes. When asked specifically about promoting spatial thinking with comics, however, we can see a certain degree of skepticism among the students (Figure 2).

Although $47 \%$ of the students favored comics for teaching geography, more than a third (34\%) of the participants are at least slightly skeptical about teaching spatial thinking with comics. Evaluating the answers to the open questions provides us with answers to why the participants believe this. More than three quarters $(78.1 \%)$ of the participants think that young learners like comics. More than two thirds $(71.9 \%)$ of the participants believe that comics are easy to understand. Statements corroborating these points of view amount to 110 out of 153 overall statements. In contrast, we counted only 23 occurrences of statements touching upon the topic of spatial thinking. Almost a third (31.1\%) of the participants even gave no answer or invalid answers showing severe difficulties reasoning for or against promoting spatial thinking with comics.

The analysis of the 3-min papers shows that, overall, some knowledge about conceptualized spatial thinking previously existed in most students, with some even displaying good results, but generally speaking their expertise was only mediocre. With four existing concepts (see Spatial Thinking in the Context of Comics Section) the participants could earn eight points by 1) mentioning and 2) explaining each concept. The average score was 5 out of 8 points, with a minimum of 0 , and maximum of 7 . We analyzed item difficulty for each point with the Rasch model, which produces difficulty values on a ratio scale for each test item. The Rasch analysis showed that it was easiest for the students to mention the spatial concepts of space as a container and space as spatial relations ( -3.73 each). Mentioning the concepts of space as a social construction and of perceived space was comparably harder $(-1.83)$. Providing definitions was harder overall than just naming the concepts. The easiest definition was the one for space as a container (1.1), followed by the ones for perceived space (1.99) and space as spatial relations (3.68). It was hardest to provide a definition for space as social construction (4.36).

To summarize our analysis of the open questions in the questionnaires, the general attitude displayed by the students was in favor of the use of comics for teaching Geography. Some students do believe that comics have the capacity to promote spatial thinking, but spatial thinking was not the main reason our participants gave for using comics in Geography classes. Before our intervention about spatial thinking, our teacher students wanted to use comics in their future classrooms because, in their view, children 


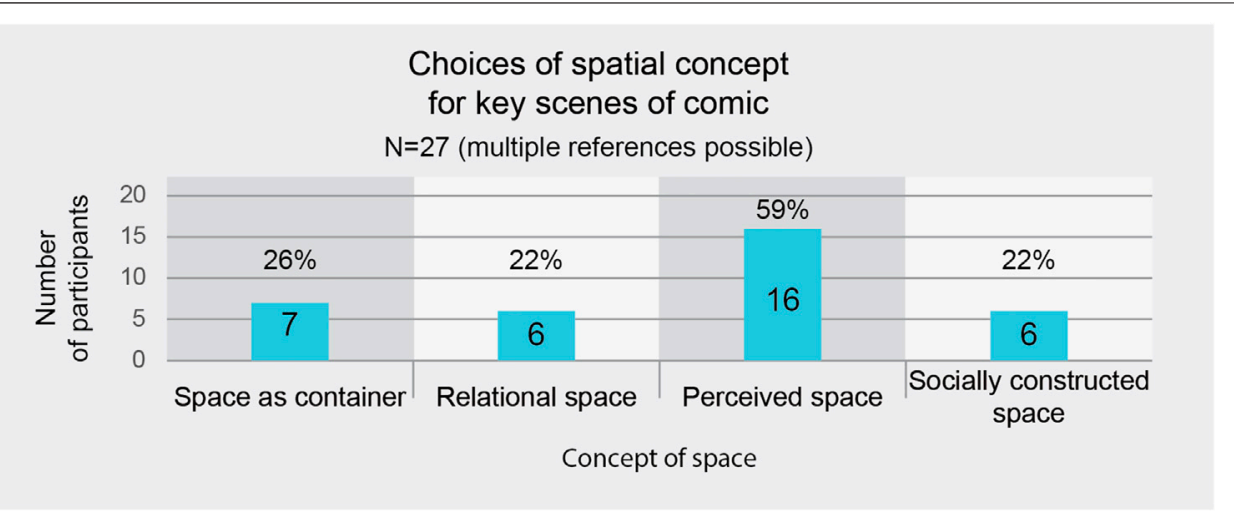

FIGURE 3 | Choices of concepts of space (after Wardenga, 2002) among the participants for their own comics.

like them and they are easy to understand, not because they are a suitable medium to convey geographical space in a meaningful way. In addition, despite of the anonymization of the questionnaires, the participants might have been influenced by the institutional setting, being in a studentteacher relationship with the researchers. It is important to note that the study took place within the context of an ongoing seminar about using comics for teaching Geography. This might have led to the participants feeling it was socially desirable to value comics.

Generally speaking, our students had difficulties to link comics with spatial thinking on a theoretical level. We would now like to contrast these findings with our participants' practical ideas and their own comics. For our analysis, the verbal utterances of the questionnaires were later complemented by the sketches and comics actually produced by the participants themselves.

\section{What are students' Practical Ideas for Translating Conceptualized Space into the Comic Form?}

Examining the questionnaires it is safe to say that after the intervention all participants were readily able to use the language of comics to express spatial thinking, at least on a theoretical level. In terms of the four spatial concepts, all of them were taken into account in the participants' comics. The most popular of the four spatial concepts among the participants for their own comics was the concept of individually perceived space (Figure 3), although this was not the easiest concept to memorize or explain, according to our Rasch analysis. However, this concept also generated the most ideas reasoning for using it or planning for its realization.

The choices of the other spatial concepts were fairly evenly distributed at mid-level amounts (22-26\%). This includes the concept of space as a social construct, which, according to the Rasch analysis, was the hardest to memorize as well as to explain. Nonetheless, the students were able to find convincing reasons for using this concept in their comics and came up with unique ideas of how to realize it.
The number of different categories and their occurrences (Table 1) show that a fairly wide range of reasons were given by the participants for using their chosen spatial concepts. Almost all the reasons expressed by the participants are convincing and thus suggest a good understanding of the concepts after our intervention (see Methods section). While some of the reasons concern the illustration of the particular perspectives of the stories' protagonists, others target the potential audience and aim to improve the reading experience by evoking emotions in the reader. The same is true for the stylistic means mentioned by the participants. Their ideas of how to express the concepts of space are abundant and diverse. They make use of many of the possibilities which comics offer in their combinations of text and image. The most common idea was to use the depiction of the characters' emotions (14 occurrences), followed by text (10 occurrences), as stylistic means. The students mainly planned on using text to express the characters' speech or thoughts. However, they developed far more visual or pictorial means of expression than textual ones: 17 out of 19 categories describe visual means of expression, while only two categories mention the use of text. Eight out of 27 participants chose a combination of two or three concepts for their comics or for single scenes. The concept of individually perceived space generated the most categories in terms of stylistic means. There were far more statements offering ideas of how to realize this concept in the comics 38) than there were comics using it (16; Figure 3). This reflects the high degree of creativity in spatial thinking within the group. Giving reasons for using a particular spatial concept was hardest for the concept of space as social construction, with three invalid statements, which were unintelligible to the researchers. This reflects the difficulty of explaining this concept, which was also shown in the Rasch analysis.

Generally speaking, Table 1 shows that the students' verbal statements show legitimate reasoning and ideas for realizing the spatial concepts proposed by Wardenga (2002) for Geography education in their comics. This suggests a good understanding of the concepts and their application. (For an analysis of students' comics with regard to their non-verbal explications, see section 4.2.2.) The analysis of the students' practical ideas 
TABLE 1 | The participants' reasoning and planned use of stylistic means for the concept of space of their choice. The table is organized according to the concepts of space, listing the participants' reasons and stylistic ideas based on the transcripts in each section.

\section{Container space}

Deductive main category

Reasons (total: 5)
Stylistic means (total: 13)
Induced subcategory

Impersonal viewpoint in storytelling

Representation of a habitat

True to life/iconic imaging

Depiction reduced to essentials
Representation of geographic reality

Occurrences of statements

Induced subcategory

Occurrences

Representation of protagonist's perspective

19

Reader's immersion in the story

Call for action on reader's side

No comment

Stylistic means (total: 38)
4

4

11
Specific depiction of character

Text

Picture (undefined)

Selection of motif

Colorization

Exaggeration

Action

Size differences

\begin{tabular}{ll}
\hline Perceived space & \\
\hline Deductive main category & Induced subcategory \\
\hline Reasons (total: 24) & Representation of protagonist's perspective \\
& Reader's immersion in the story \\
& Call for action on reader's side \\
Stylistic means (total: 38) & No comment \\
& Specific depiction of character \\
& Text \\
& Picture (undefined) \\
& Selection of motif \\
& Colorization \\
& Exaggeration \\
10 & Action \\
3 & Size differences
\end{tabular}

Space as network of spatial relations

Deductive main category

Reasons (total: 5)

Stylistic means (total: 10)
Induced subcategory

Occurrences

Depiction of two or more scenes of action at once

No comment

Specifically arranged elements of reality

Text

Visual metaphor

Placement of speech bubbles

Movement
4

4

4

3

1

Space as social construction

Deductive main category

Reasons (total: 16)

Stylistic means (total: 9)

\section{Induced subcategory}

Display of clichés in the story

Heightened familiarity for the reader

Display of disparities

Unconvincing

Symbols or symbolic objects

Dividing visual elements

Colorization
Occurrences

\section{7}

4

2

3

7

1 shows a wide array of different ideas (Table 1), many of which were clearly not sourced from our intervention (Methods Section). A comparison of the participants' ideas, listed in Table 1, with the content of our intervention reveals just how creatively the participants have performed their task (Figure 4): a large share of the students' statements cannot be found either in our review of the spatial concepts or in the example comic pages provided by us to illustrate possible realizations of the concepts. From this we can conclude that many of the ideas the participants expressed were inspired by their own creations of scenes in their plots (Figure 4). The students produced these ideas in the process of thinking up their comic projects, which were clearly related to the students' own creativity in spatial thinking.

\section{How do the Finished Products Relate to the Students' Theoretical Understanding of Conceptualized Space?}

The analysis of the students' verbally expressed spatial thinking showed a good understanding of the spatial concepts. Do the sketches and finished comics corroborate that view? We interpreted the comics on the grounds of the students' choices of spatial concept, which they communicated in the 


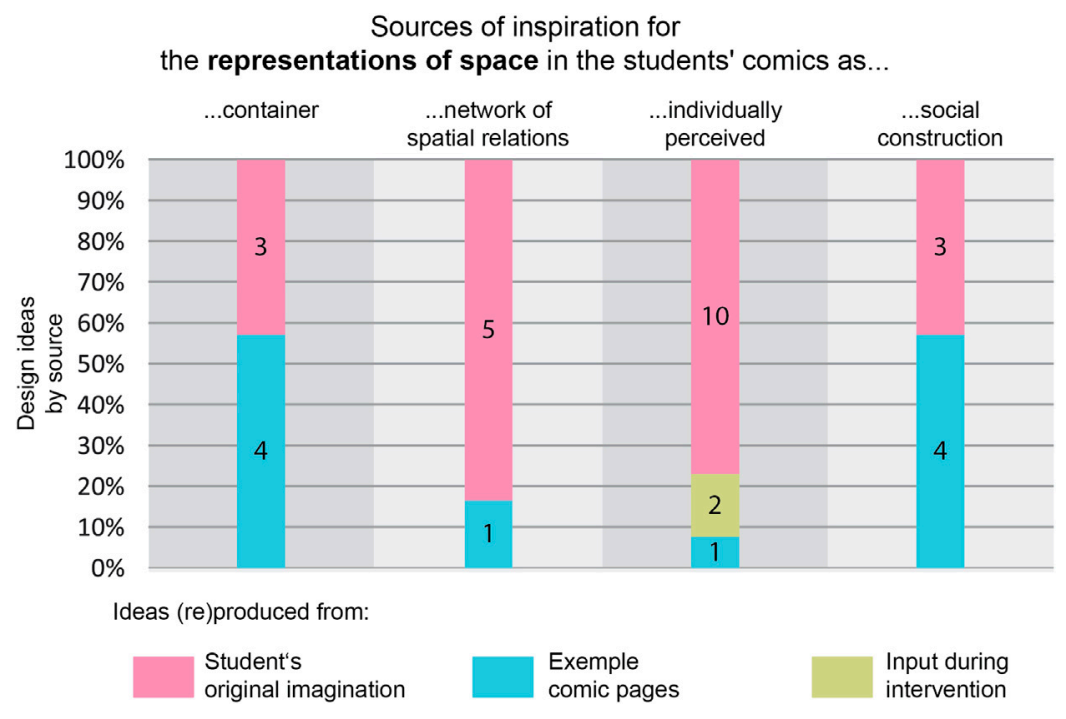

FIGURE 4 | Creation (pink) and reproduction (blue and green) of ideas by the participants for the representations of space in their own comics. $N=27$.

questionnaires. Under this prerequisite the analysis is surprisingly fruitful in revealing a deep level of understanding-or interpretation-of the concepts of space. Another interesting phenomenon is that many students mix the concepts of space in their stories. Eight out of the 27 analyzed comics were planned from the beginning to combine different concepts, sometime even in one single panel or scene. In most of the comics we observed a mix of the concepts of space as a container, perceived space, and space as a social construct.

\section{Space as Container in the Students' Comics}

Seven students chose this concept for their comic stories (Figure 3). Most of them wanted to address an authentic setting in an objective way. One person who chose this concept compared living conditions in rural and urban areas in their comic. The drawings of this person are very reduced, showing only the rough shapes of certain features associated with built up urban areas versus rural areas, such as high rise buildings and flat ground for urban areas, whereas rural areas are depicted with small houses and a tree. Both prototypical landscapes are identified using text, which the author lays out as headlines above the drawings. In this example we can see very well that the more reduced a drawing is the more it represents the ideas of the object it represents, rather than a thing that actually exists in the real world. This then is closer to the collective imagination of things and thus places, represented by symbols almost like in a map. In drawing from memory the author also taps into his or her own repertoire of imaginations, which are influenced by collective social construction. In this case the creator thus referred to social constructions of space via their own perception of space. The use of oversimplified shapes raises the question of whether the concept of space as container is as easily realized in the comic form as might have been anticipated by the students. After all, the Rasch analysis demonstrates that this was the easiest of the four concepts in terms of memorizing and defining it. In fact, this example illustrates the difficulties that any comic creator encounters when attempting to describe reality. As table 6 shows, the students in this study prefer to use this concept with an underlying realistic understanding of geographical space to address real phenomena in the real world. Due to the fabricated nature of drawings in general, the students have to think about how reality should be represented in their comics, taking into account that readers are supposed to recognize their drawings, forcing them to tap into the societal discourse of what is real. All of a sudden they have to think about what are generally considered to be the essential features of a given object, quickly leading to the use of well-known stereotypes as a shortcut to a general understanding in the target group.

In order to avoid this difficulty of slipping off into the realm of socially constructed symbolism, another participant tried to make his representations of places as specific as possible: they added rain to a scene depicting the living conditions of a family with the goal of "intensifying the authenticity" of the scene. This student also tried to authenticate the scene by using a bird's eye perspective and a signpost indicating a location with text. Using text not as a symbol or representation of speech but turning the text into a part of the image is an interesting strategy unique to visual narratives. It seems that the author attributes a more persuasive power to pictures than text where the representation of reality is concerned. Text is also used for authentication in another comic, where objects are labeled with brand names like Porsche and Gucci. This reference to existing entities marks the objects as being part of the real world. This reminds us of Magritte, although in this case the technique was probably meant to compensate for a lack of ability to draw photo-realistically.

Another strategy to counter this difficulty of slipping into the concept of social construction is to use photography instead of 


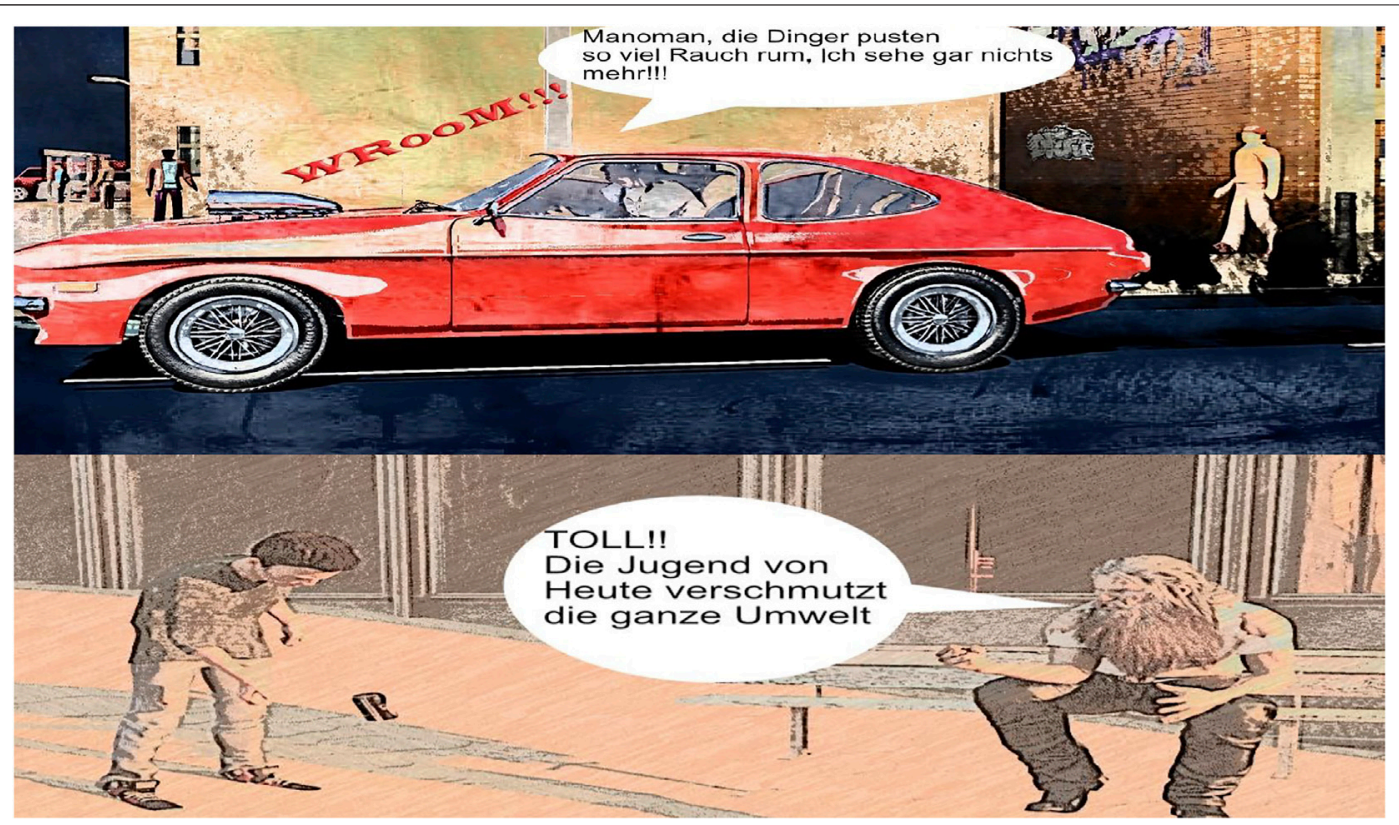

FIGURE 5 | Using photographs for a convincing representation of a possible world. The author aims at representing geographical space as a container while at the same time merging it with perceived space. The text in the speech bubbles reads: "Wow, those things produce so much smoke, I can hardly see anything!!", and "Just perfect! The kids nowadays pollute the entire environment". Image courtesy of M. Adolph.

drawn imagery in the comic (Figure 5). The presumed objectivity of photographs is used to convince the audience of the plausibility of the story world. The story is about a possible world, a kind of dystopia reaching into the future. Photographs suggest a high degree of specificity, making them appear credible and related to the real world, as demanded by the concept of space as a container. At the same time, photographs are very able to capture moods and thus individual perceptions of environments. In one story the student, like three other participants in this study, attempted to merge the concepts of container and perceived space, which is a difficult task, given that they are on opposite sides on the spectrum of objectivity. To accommodate this contradiction, the author processed the photos in color and contrast so that they conveyed slightly surreal impressions. This produced a representation not of a factual, but of a possible world. The story looks into the future under the premise that certain processes, which exist today in the real world, will continue into the future. The result is a projection of a world that might exist under certain realistic assumptions.

The students' comics show that they generally seemed to be aware of the difficulties they encountered when using the concept of space as a container in comics, which seemed to be the easiest concept according to the Rasch analysis. The tension between a serious attempt at depicting geographical space as objectively as possible on the one hand, while, on the other hand, deploying the socially constructed imaginations of reality which we only accept as true, offers the opportunity for a rewarding reflection in the classroom. It is exactly these kinds of struggles which can be used to discuss with the students issues of geographical truth and representation. This raises awareness of the degree to which our ideas of space and the real world are constructed.

\section{Space as a Network of Spatial Relations in the Students' Comics}

The participants who had chosen this concept of space came up with very interesting comic related ideas, using the space on the page to arrange their drawings, panels or text elements, e.g. juxtaposing pictures with opposing orientation at the top and bottom of the page. However, a difficulty seemed to be to link the creatively used space of the page to geographical space in the real world. The intricate page layout did not always represent spatial relations in a geographical sense. Another difficulty occurred when the concept chosen needed to show two processes taking place simultaneously at different locations. Although the participants used typical features of image composition to express spatial relations such as perspective, or contrasts of foreground and background, big versus small, aggressive versus peaceful, and so on, these were rarely relevant for the geographical problem discussed in the story. Another example is the juxtaposition of the same space at different times. Here a timely relationship is expressed using the space of the page. This is typical of comics as a sequential art. But the page space does not automatically express spatial relations, so these attempts failed to display spatial relations while focusing on temporal relations. Another case shows a juxtaposition of two locations where the story takes place, the school and the home. However, no relevant relationship between them is subject of the narrative. In some cases the participants displayed in their comics the emotional relationships of stakeholders making 


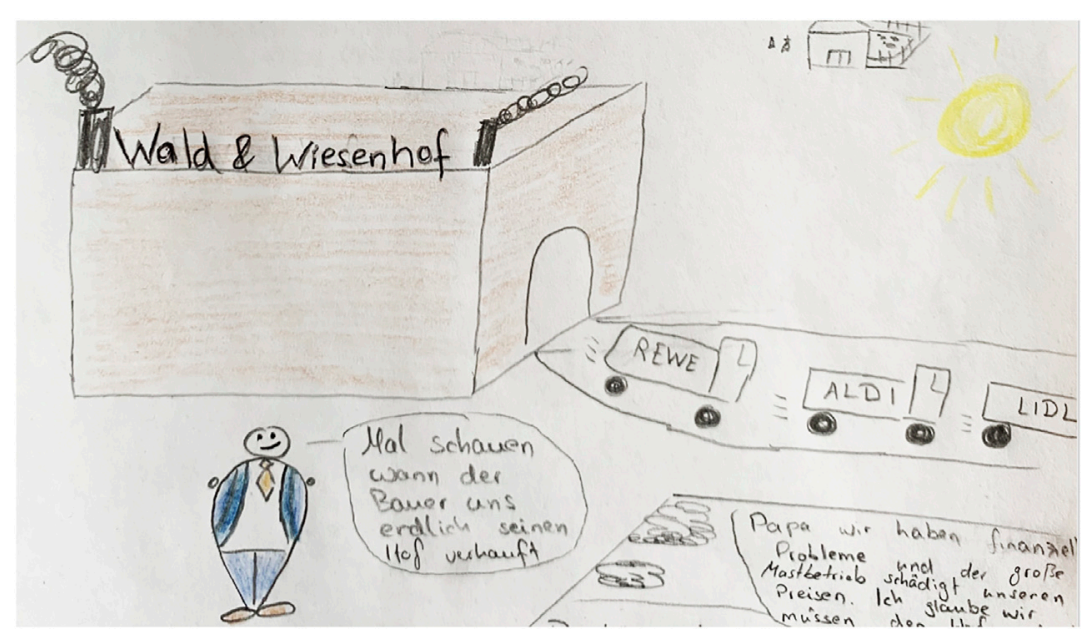

FIGURE 6 | Emotional link between a small and a big farm sharing a common space. Spatial relations resonate in this image but the author does not make them explicit. The text in the image reads as follows: "Country Farm", names of supermarket chains on the trucks, "Let's see when the farmer finally sells his farm.", "Daddy, we have financial issues, and the huge pig mast farm is destroying our prices. I'm afraid we have to sell our farm." Image courtesy of D. Lux.

use of a shared space, relating them back to what happens in these spaces.

Figure 6 shows the key scene from a comic dealing with rural development. In the background we see a traditional farm, which is metaphorically pushed aside by an industrial pig mast farm. The owner of the pig mast farm displays a contented facial expression, while the owners of the traditional farm express their worries about this new competitor in a text bubble in the lower right corner. By using image composition in these ways, the authors mix in a lot of the emotions which might be associated with the concepts of perceived space. However, in this case, the small farmers' individual perception of what is happening right next to them-which represents a spatial relationship-influences their decisions about what to do with their land. The creator here uses individual perception to express the quality of a spatial relationship by means of image composition, emotional character design and text.

We can conclude that one major difficulty seemed to be to discriminate between spatial and other relationships between objects sharing a space. Some of our participants, seemingly intuitively, assigned more importance to emotional relations than to spatial relations. Interestingly the heated emotions often do result from the protagonists' sharing the same space in the comics, so that a relevant spatial relationship between the parties often does exist in the story world. However, some authors struggled with identifying the underlying spatiality of a conflict, revealing a need to reflect more on spatial thinking. In the classroom this offers an opportunity to identify the specifically geographical factors of conflicts and, through this, practice spatial thinking. During the production of the comics, the teacher can for instance encourage students to incorporate maps into their comics. This can be done when the comics are finished, as well. Each participant in the learning could draw a map of the story world. They can then compare their maps and also discuss issues of perception where the maps differ. This helps to single out the spatial aspects of a conflict, which might also produce emotional reactions.

\section{Individually Perceived Space in the Students' Comics}

The vast majority of participants chose to represent space as it is subjectively perceived by the protagonists in their comics. It seems noteworthy that four out of the sixteen people who had chosen this concept attempted to combine it with the concept of space a container, which we discussed above. Reasons for this might be that they try to emphasize the plausibility of a possible reality, or that they want to express the ambiguity of the subjective perception of an objectively existing phenomenon. This seems to be a hard task, as some of their preparatory sketches prove.

Most participants expressed the personal view of their protagonists by displaying the characters' emotions. Even in the crudest of sketches we can identify at least some basic emotionality in the characters' faces. More advanced drawings also display emotional body language. Several examples show how a character's emotions are directly linked to a place by presenting them in a specific location. Other creators use the possibilities of drawing landscapes to show a protagonist's viewpoint. One student tells a story of the protagonist's development from disliking the city of Tokyo to becoming quite fond of it at a later stage. She depicts these two contrasting stages using different motifs and thus creating moods. She creates the estranged feeling of the protagonist, rooted in his dislike, by using angular shapes, a wide shot camera angle from a position far away, no natural shapes, straight lines, empty spaces without people or other living beings. When the protagonist has grown to like the city, she depicts him in a park or garden accompanied by a person, surrounded by round, organic shapes, and in places offering comfort, like a bench to sit on, a pavilion with a roof; there are plants and the calm water of a small pond. Another student uses an expressive brush stroke to place his protagonist in the chaos he is perceiving at this moment (Figure 7). There are objects 


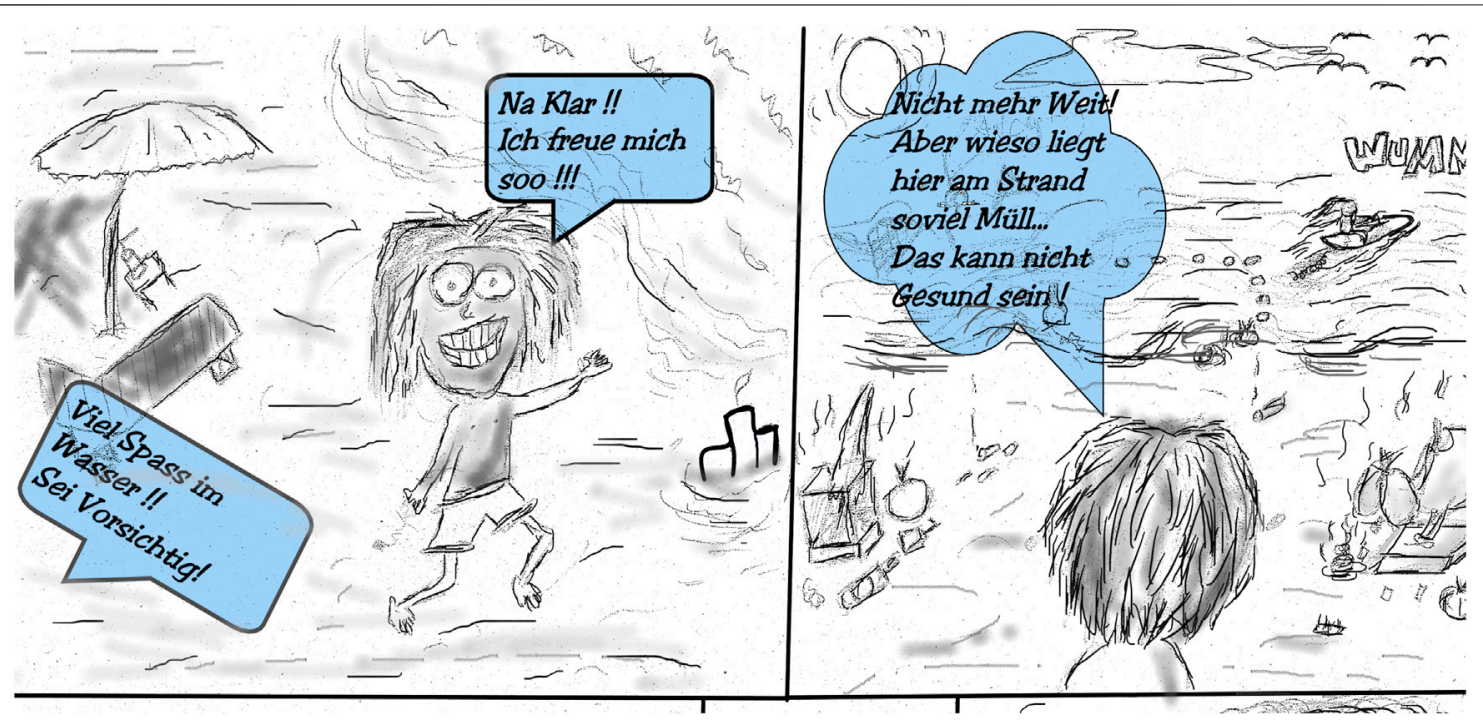

FIGURE 7 | Using an expressive drawing style to represent the subjective perception of space. In the second panel the reader should take on the perspective of the protagonist. The text in the speech bubbles reads as follows: "Have fun in the water!! Be careful!", "Sure thing!! I'm so excited!!!", "Almost there! But why is there so much garbage lying around at this beach ... This can't be healthy!" Image courtesy of H. Tunc.

representing trash scattered all over the polluted beach, blurred shapes, wiggly lines. The author also uses a tilted speech bubble, referring to a speaker out of the sight of the audience, to add to the chaos. This panel thus represents an outside perspective. In comics, the perspective can change from one panel to another using the gutter between panels. The gutter forces the reader to construct the story in his or her own head. This engagement of the reader can bridge wide gaps in storytelling. This very peculiar capacity of comics was deployed by the author in the following panel, changing from an outside to an inside perspective. The position of the protagonist in the second panel of Figure 6, in particular, tells us whose perspective we, as readers, should follow from here. The "camera" is positioned behind the protagonist so that it almost seems that we see the world "through his eyes".

In contrast to these examples, which use a mostly non-verbal way of communicating emotion, other examples show that emotional story telling might also take place in the textual part of the comic. Sometimes the authors use speech or thought bubbles to link the emotions displayed in the drawing with the geographical topic in question by means of text. They express dismay or approval, for example, verbally through the characters' direct speech.

All in all the participants using this concept of space were mostly successful in putting their ideas to practice. However, in the context of comic production, we could observe that it seemed difficult for many students to acknowledge their own subjectivity when trying to represent places in their stories. The comics shed light on some of the students' understanding of the existence of multiple perspectives in a way which is impossible for textual descriptions alone. A perspective in this sense seems to be a particular view on a shared reality. This explains why text is a preferred stylistic means for some participants to express perceptions of space. They used speech bubbles attached to characters to express personal feelings and viewpoints about the world as it is perceived, while they use the image parts of the comic for depicting the world "as it is". Based on this understanding of perspective the teacher can discuss the difficulties of representing the world objectively by introducing the perspective of the authors, which the students hold themselves. By making visible the subjective nature of the students' drawings, the teacher can initiate a process of self-reflexivity in and with the students. This could lead to a discussion of how to deal with this lack of objectivity, e.g. in science, news media etc.

\section{Space as Social Construction in the Students' Comics}

It was predominantly students who were dealing with topics such as prejudices in their comics who chose to use the concept of space as social construction. Many reasoned that they wanted to display clichés or stereotypes in their stories. This worked best in those stories which subsequently questioned those stereotypes.

A good example of successfully dealing with this concept of space is a comic about segregation (Figure 8). The story spatializes social and psychological prejudices, connecting them with the places of the protagonist's origin. This is echoed in the key scene of the comic, which has a dividing line between very differently colored and equipped representations of two neighborhoods. In the comic, the author also played out the differences of socially constructed versus individually perceived space, with the protagonist perceiving places in terms of the imaginations of a certain social group. The protagonist comes from a "bad area" in his city but attends school in a rich neighborhood. The rich kids use this to make fun of him, which saddens the protagonist. Later he returns to his area and finds pride in its unique cultural places, like the basketball court or a graffiti wall. In this way the author deconstructs the previously introduced social constructions of space. Finding his strengths, the protagonist breaks with the socially constructed "reality" of his inferior identity, which was imposed on him by 


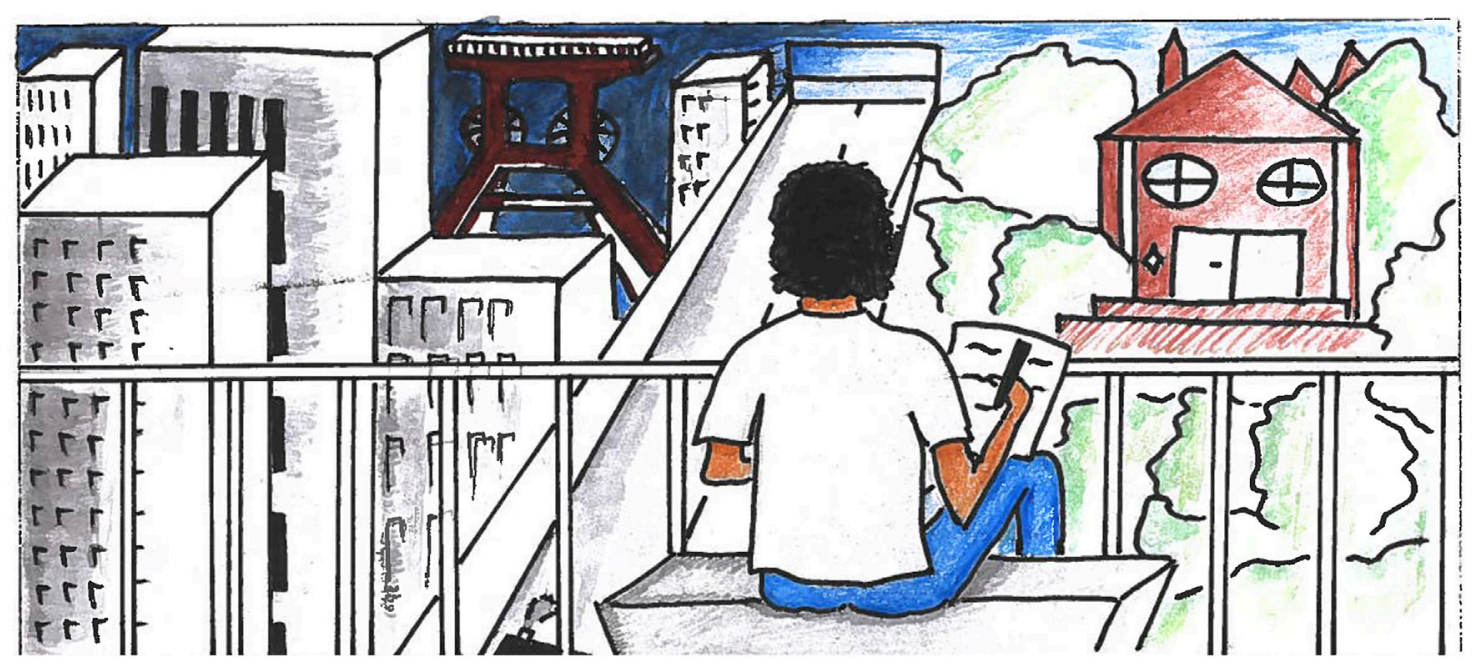

FIGURE 8 | Depicting the social construction of space and questioning it by adding a personal view on it. Image courtesy of J. Becker.

others and was also accepted by him. The collective imaginations shared by a social group in power and imposed on the protagonist are breached by recurring to the subjective perception of space through the protagonist's eyes. In this and other stories the tension created by contrasting social constructions of space with a personal perception provides the opportunity to critically examine, in the classroom, the pitfalls of collective imaginations, exposing them as constructs that are not necessarily true. Both comics also reveal a very deep understanding of the concepts of space, which we could not identify in the verbal expressions of the questionnaires from the planning phase. Clearly it was the making of the comic story which brought this to light.

However, there were difficulties, too. One student uses a picturein-picture technique, depicting the protagonists looking at a poster on the wall. The poster displays a polar bear floating on a small iceberg. This is a reproduction of a commonly used trope in factual news stories about climate change. Using a picture within a picture is a very effective way of referring to space as a social construction, separating it from the actual story world. However, the author does not use the opportunity to take a critical stance towards this generalized imagination. The student rather uses it to construct the moral message of the story, which is that we should protect our climate. So, willingly or unwillingly, the author ends up using socially constructed imaginations to persuade the audience, when the goal should be to empower learners through critical media competencies.

In summary, we observed very successful approaches to socially constructed space, which was the hardest of the concepts to memorize and explain according to the Rasch analysis. In some cases difficulties occurred in unmasking the social constructs. Even in cases where students clearly announced in the questionnaires that they would use social constructions to represent their story world spaces, they sometimes hesitate to deconstruct them. In those cases where collective imagination are used as a means of persuasion and are not revealed and critically questioned by the students, educators can use the comics as the perfect occasion to shed a critical eye on the workings of social constructs in comics and in the media in general. In this sense, the creation of the comics revealed a unique opportunity to deepen this understanding and achieve a self-reflexive stance in the learners.

\section{DISCUSSION}

This study shows that, even though initially it might not be obvious for learners to link comics with spatial thinking, the visuo-verbal language of comics can help to express ways of spatial thinking and provide deeper insights than verbal utterances alone. Generally speaking, our students welcomed comics as a means of teaching, despite the fact that they at first had difficulties exploiting the medium for spatial thinking. Teaching spatial thinking with comics has distinct advantages, as we were able to demonstrate in this study. Producing their own comics, our students developed a vast array of diverse and creative practical ideas, thus enhancing their spatial thinking by expanding their ability to express conceptualizations of space in more than just verbal modes. Comics can acommodate visual representations, which are after all, according e.g., to Elbay (2020), highly important in the process of spatial thinking.

Our survey corroborates the motivational effect of learning with comics reported by Kilıçkaya and Krajka (2012) in their scientific study on teaching language with comics, as well as the work of Lay (2021), who describes an example of best practice with international school children creating their own comics. One main reason our participants welcomed the idea of teaching geography with comics stemmed from the assumption that comics are easy to understand. This is also stated by Gomez (2014) and Kleeman (2006), who scientifically examine comics as learning materials in geography. They claim that comics can break learners' resistance to dealing with complex topics. Lin et al. (2015) are another example of researchers who assume that comics are easy to understand. In the light of this study we would like to qualify this claim and rather state that comics make 
complex matters more accessible with their use of different medial channels such as text and pictures. In this way, educators can induce valuable reflections in the learners, promoting a deep understanding of geographical subject matter and spatial thinking.

In effect, we would like to emphasize that the benefits of teaching spatial thinking with comics exceed the mere fun factor of consuming supposedly easy to understand media. Although it might be fun for some student teachers to learn with comics, we found that even in large groups there might only be a very few persons who are interested in or fond of comics. This is contrary to the assumption that young learners generally like comics. So if we examine the benefits of comics for teaching Geography, we have to take the medial capabilities of comics into account, and not the popularity of the medium, which is probably rooted in content, e.g. superhero stories. We therefore focused on learning processes and, like da Silva et al. (2017) or Elsayed and Daif (2019), observed a high degree of creativity in the students when they had to produce their own comics. This creativity was triggered by the application of theoretical knowledge to concrete situations, which enhanced the students' conceptual spatial thinking capabilities. Comparing the input of the intervention with the students' verbalized output in the questionnaires we can observe that they produced many of their ideas for representing space by themselves in the process of conceiving and planning their own comic stories. Their ideas for expressing spatial concepts were abundant and convincing. This might be the result of an immersion in the visual and the verbal aspects of space through the concrete situations of their stories, which they had to think up on their own. Additionally, the production of a story and thus the fabricated nature of comics as such seem to lead to a deeper understanding of how space might be constructed and perceived.

Our study shows, for the first time, that learners can express spatial thought processes much more exhaustively with comics than with text alone. Spatial thinking is an integral and key aspect of Geography education. In the comic form, learners can tackle spatial conceptions and geographical problems from more angles and using different media channels than when using verbal expression alone, allowing for a deep understanding of the subject matter from a geographical stance. At the same time, our students-being future teachers themselves-learned a lot about how comics work as a medium. This prepared them to use comics in their own classes in a qualified and critical way. Another important factor in using comics for learning about space is the storytelling aspect of the medium. A story requires the reader to take a perspective, mostly that of the protagonist. Perspective in its metaphorical as well as literal sense (as required in a visual medium such as comics) makes the meaning of spaces and places visible. We can take on a scientific perspective, the perspective of users of space, or of decision makers and so on, each of which has its counterpart in its pictorial realization. Having to mix and match these aspects, our students were pressed to think their concepts through.

The participants in our study demonstrated that the versatility of comics can very well be put to use in expressing and triggering spatial thinking. Additionally, the production process and the final comics themselves unfolded the students' thought processes for the teachers. As such, they are a very important resource for uncovering misunderstandings, challenges, or misconceptions. The necessity to express oneself both verbally and visually leaves less space for hiding or covering gaps in knowledge. Creating a convincing comic story means thinking things through thoroughly, which evidently reveals any inconsistencies, leading in turn to more reflection. Creating contrast can similarly cast light on conflicting ideas. For instance, one participant planned to visualize an accurate representation of space in the panels while contrasting it with the protagonist's individual perceptions in the speech bubbles. The very idea of using the different modes of the medium to express different concepts of space is intriguing. Using parallel channels for contrasting spatial concepts can create a welcome tension in the reader. The teacher can then use this tension to question and explore the topic in question in more depth (Rhode-Jüchtern, 2015). This example shows how comics can easily host very complex thoughts about conceptualizations of space.

This complexity, however, might have been underestimated by the participants, due to their assumption that comics are an easy to understand medium. In comics as teaching materials, we have to accommodate pedagogic demands, storytelling, scientific soundness and graphic representation at the same time. This is highly demanding. Students should therefore team up when producing the comics, so that their talents can complement each other and they can share tasks and support each other. It is also quite important to point out that a guided reflection should accompany the process to induce the reflection of space, which is according to Rhida et al. (2020) constitutional to spatial thinking. Only if we help the learners to step back and think about how they can express spatial assertions with comics can they achieve a deep understanding of spatial concepts. They can then turn their spatial thinking into a "distinctive form of reasoning", as Anthamatten (2010, p. 169) calls it. We recommend explicit instructions for reflection both during and after the creation of the comics. While preparing the plot and basic ideas for the comics, the learners should be constantly reminded to think about how they fit into geographical conceptions of space. They should be explicitly asked to decide which of the spatial concepts best suits their stories, so that they explore and make use of the possibilities to express space in geographically meaningful ways. Students should define their goals and be able to explain how they intend to meet them. This allows teachers to reflect on the finished comics with the learners within the context of welldefined criteria of spatial thinking. Teachers can inquire about what ideas have led to certain portrayals of space, and the reasons behind the students' decisions. Just as comics reveal differing perspectives on geographical phenomena (Gomez, 2014), this very need to take a particular perspective while producing comics lends itself to the sparking of a discussion about the possibility of objectivity in contrast to distinct points of view. The experience of creating a comic world is an eye opening method to demonstrate the fabrication of space.

Conceiving and planning representations of spatial concepts in the comic form promotes a thorough understanding of geographical spatial thinking. Hard to understand topics can be discussed based on the learner's own experience, such as the 
relationship between media and reality, the construction of scientifically accurate representations, or the difficulties of communicating data and facts. All one needs is a pen and paper.

\section{CONCLUSION}

The results of this study suggest that our pre service teacher students profited in a twofold manner from producing their own geography related comics. First, they engaged in a deep learning process. Second, they got familiar with an innovative teaching method, which they can use themselves in their later career. In the geography class, creating comics can lead to a rich discussion and reflection on thought processes which are usually hidden behind a wall of words. We conclude that comics are a valuable if little explored teaching method for spatial thinking. Using images in combination with text triggers creative thinking and allows learners to embrace spatial concepts in a more holistic approach than just words

\section{REFERENCES}

Anthamatten, P. (2010). Spatial Thinking Concepts in Early Grade-Level Geography Standards. J. Geogr. 109 (5), 169-180. doi:10.1080/ 00221341.2010 .498898

Cohn, N. (2014). The Visual Language Fluency Index: A Measure of "Comic reading Expertise Visual Language Lab: Resources. Available at: www. visuallanguagelab.com/resources.html (Accessed on April 15, 2021).

Dallacqua, A. K., and Peralta, L. R. (2019). Reading and (Re)writing Science Comics: A Study of Informational Texts. Read. Teach. 73 (1), 111-118. doi:10.1002/trtr.1801

Deutsche Gesellschaft für Geographie (DGfG) (2014). Bildungsstandards im Fach Geographie für den Mittleren Schulabschluss. 8th ed. Bonn, Germany: Selbstverlag Deutsche Gesellschaft für Geographie (DGfG).

Disney Enterprises (2015). Die Ducks in Deutschland, Story and Art: Gulbransson J. Hamburg, Germany: Ehapa.

Elbay, S. (2020). A Foundational Perspective for Spatial Thinking in Relation to Social Studies Curriculum and Middle-School Textbooks in Turkey. Rev. Int. Geographical Educ. (Rigeo) 10 (2), 30-57. doi:10.33403/rigeo.616984

Elsayed, K., and Daif, R. (2019). Comics: A Learning Strategy for Developing Innovative Thinking of Tourism and Hospitality Under-graduate Students. J. Tourism Sports Manage. (Jtsm) 1 (2), 59-74.

Gersmehl, P. J., and Gersmehl, C. A. (2007). Spatial Thinking by Young Children: Neurologic Evidence for Early Development and "Educability". J. Geogr. 106, 181-191. doi:10.1080/00221340701809108

Gomez, C. (2014). Teaching Physical Geography at university with Cartoons and Comic Strips: Motivation, Construction and Usage. N. Z. Geog. 70, 140-145. doi:10.1111/nzg.12053

Kleeman, G. (2006). Not just for Fun: Using Cartoons to Investigate Geographical Issues. New Zealand Geographer. 62, 144-151. doi:10.1111/j.1745-7939.2006.00057.x

Kilıçkaya, F., and Krajka, J. (2012). Can the Use of Web-Based Comic Strip Creation Tool Facilitate EFL Learners' Grammar and Sentence Writing? Br. J. Educ. Technol. 43 (6), E161-E165. doi:10.1111/j.1467-8535.2012.01298.x

Lay, T. (2021). Ein Comic geht um die Welt. Available at: https:// fremdsprachedeutschdigital.de/download/fd/FD-63-Online-Lay.pdf (Accessed April 15, 2021).

Lefèvre, P. (2009). "The Construction of Space in Comics," in A Comics Studies Reader. Editors J. Heer and K. Worcester (Jackson: University Press of Mississippi), 157-162.

Lin, S.-F., Lin, H.-S., Lee, L., and Yore, L. D. (2015). Are Science Comics a Good Medium for Science Communication? the Case for Public Learning of Nanotechnology. Int. J. Sci. Educ. B 5 (3), 276-294. doi:10.1080/21548455.2014.941040

Mateu-Mestro, M. (2010). Framed Ink. Culver City, CA: Design Studio Press. alone. Teachers can profit from this method, too, as they can diagnose the strengths and weaknesses of learners based on more aspects than verbal expressions alone.

\section{DATA AVAILABILITY STATEMENT}

The raw data supporting the conclusion of this article will be made available by the authors, without undue reservation.

\section{AUTHOR CONTRIBUTIONS}

FR and $A B$ contributed to conception and design of the study. FR collected and analysed the data, Interpretation of data: FR and $\mathrm{AB}$. Writing- original draft preparation: FR, WritingReview and Editing: FR and AB, supervision: $A B$. All authors contributed to manuscript revision, read and approved the submitted version.

Mayring, P. (2014). Qualitative Content Analysis: Theoretical Foundation, Basic Procedures and Software Solution. Available at: https://www.ssoar.info/ssoar/handle/document/39517 (Accessed April $15,2021)$

Munier, G. (2000). Geschichte im Comic: Aufklärung durch Fiktion? über Möglichkeiten und Grenzen des historisierenden Autorencomic der Gegenwart. Hannover, Germany: Unser Verl.

Rhode-Jüchtern, T. (2015). Kreative Geographie. Frankfurt am Main, Germany: Wochenschau Verlag.

Ridha, S., Putri, E., Kamil, P. A., Utaya, S., Bachri, S., and Handoyo, B. (2020). The Importance of Designing GIS Learning Material Based on Spatial Thinking. IOP Conf. Ser. Earth Environ. Sci. 485, 012027. doi:10.1088/1755-1315/485/1/ 012027

Schüwer, M. (2008). Wie Comics erzählen. Grundriss einer intermedialen Erzähltheorie der grafischen Literatur. Trier, Germany: WVT.

Silva, A. B. D., Santos, G. T. D., and Bispo, A. C. K. D. A. (2017). The Comics as Teaching Strategy in Learning of Students in an Undergraduate Management Program. Ram, Rev. Adm. Mackenzie 18 (1), 40-65. doi:10.1002/ trtr.180110.1590/1678-69712017/administracao.v18n1p40-65

Tatalovic, M. (2009). Science Comics as Tools for Science Education and Communication: a Brief Exploratory Study. J. Sci. Commun. Jcom. 08 (94), 1-17. doi:10.22323/2.08040202

Wardenga, U. (2002). Alte und neue Raumkonzepte für den Geographieunterricht. Geographie heute 200, 8-11.

Witek, J. (2009). “The Arrow and the Grid," in A Comics Studies Reader. Editors J. Heer and K. Worcester (Jackson: University Press of Mississippi).

Conflict of Interest: The authors declare that the research was conducted in the absence of any commercial or financial relationships that could be construed as a potential conflict of interest.

Publisher's Note: All claims expressed in this article are solely those of the authors and do not necessarily represent those of their affiliated organizations, or those of the publisher, the editors and the reviewers. Any product that may be evaluated in this article, or claim that may be made by its manufacturer, is not guaranteed or endorsed by the publisher.

Copyright (C) 2021 von Reumont and Budke. This is an open-access article distributed under the terms of the Creative Commons Attribution License (CC BY). The use, distribution or reproduction in other forums is permitted, provided the original author(s) and the copyright owner(s) are credited and that the original publication in this journal is cited, in accordance with accepted academic practice. No use, distribution or reproduction is permitted which does not comply with these terms. 